\title{
Initiation of Pharyngeal Swallow with Bolus Head a Posterior Laryngeal Surface of Epiglottis
}

National Cancer Institute

\section{Source}

National Cancer Institute. Initiation of Pharyngeal Swallow with Bolus Head a Posterior Laryngeal Surface of Epiglottis. NCI Thesaurus. Code C127222.

A finding of pharyngeal swallow initiated with bolus head at posterior laryngeal surface of epiglottis. 